\title{
BMJ Open Role of IgM testing in the diagnosis and post-treatment follow-up of syphilis: a prospective cohort study
}

\author{
Kara K Osbak, ${ }^{1,2}$ Achilleas Tsoumanis, ${ }^{1}$ Irith De Baetselier (iD , ${ }^{1}$ \\ Marjan Van Esbroek, ${ }^{1}$ Hilde Smet, ${ }^{1}$ Chris R Kenyon, ${ }^{1,3}$ Tania Crucitti (D) ${ }^{1}$
}

To cite: Osbak KK, Tsoumanis A, De Baetselier I, et al. Role of IgM testing in the diagnosis and post-treatment followup of syphilis: a prospective cohort study. BMJ Open 2020;10:e035838. doi:10.1136/ bmjopen-2019-035838

- Prepublication history for this paper is available online. To view these files, please visit the journal online (http://dx.doi org/10.1136/bmjopen-2019035838).

KKO and $A T$ contributed equally. CRK and TC contributed equally.

Received 19 November 2019

Revised 23 June 2020

Accepted 06 August 2020

Check for updates

(c) Author(s) (or their employer(s)) 2020. Re-use permitted under CC BY-NC. No commercial re-use. See rights and permissions. Published by BMJ.

${ }^{1}$ Department of Clinical Sciences, Institute of Tropical Medicine, Antwerp, Belgium

${ }^{2}$ Department of Medical Microbiology and Infectious Diseases, Erasmus University Medical Center, Rotterdam, The Netherlands

${ }^{3}$ Department of Medicine, Groote Schuur Hospital, Observatory, South Africa

Correspondence to

Dr Tania Crucitti; tcrucitti@itg.be

\section{ABSTRACT}

Objectives The diagnosis of repeat syphilis and its followup remains challenging. We aimed to investigate if $\lg \mathrm{M}$ testing may assist in the diagnosis of syphilis reinfection/ relapse and its treatment follow-up.

Methods This substudy was conducted in the context of a syphilis biomarker discovery study (ClinicalTrials. gov Nr: NCT02059525). Sera were collected from 120 individuals with a new diagnosis of syphilis (72 with repeat infections) and 30 syphilis negative controls during a cohort study investigating syphilis biomarkers conducted at a sexually transmitted infection/HIV clinic in Antwerp, Belgium. Syphilis was diagnosed based on a simultaneous positive treponemal and non-treponemal assay result and/ or positive serum PCR targeting polA. Specimens collected at visit of diagnosis, and 3 and 6 months post-treatment were tested by two enzyme immunoassays (EIAs), recomWell (Mikrogen; MI) and Euroimmun (EU), to detect anti-treponemal IgM. Baseline specimens were also tested for anti-treponemal IgM using a line immunoassay (LIA) recomLine (MI). Quantitative kinetic decay curves were constructed from the longitudinal quantitative EIA results. Results An overall sensitivity for the diagnosis of syphilis of $59.8 \%$ (95\% Cl: $50.3 \%-68.7 \%), 75.0 \%$ (95\% Cl: $66.1 \%-82.3 \%$ ) and $63.3 \%$ (95\% Cl: $54.8 \%-72.6 \%$ ) was obtained for the EU, MI EIAs and MI LIA, respectively. When only considering repeat syphilis, the diagnostic sensitivity decreased to $45.7 \%$ (95\% Cl: 33.9\%-58.0\%), 63.9\% (95\% Cl: $51.7 \%-74.6 \%)$ and $47.2 \%$ (95\% Cl: $35.5 \%-59.3 \%$, respectively. IgM seroreverted in most cases 6 months after treatment. Post-treatment IgM concentrations decreased almost $30 \%$ faster for initial syphilis compared with repeat infection. The IgM EIAs and IgM LIA agreed from fairly to moderately (Cohen's kappa ( $\kappa)$ : 0.36 (EU EIA);

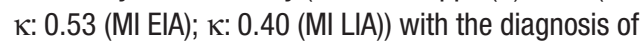
syphilis.

Conclusions IgM detection was not a sensitive method to diagnose syphilis and was even poorer in the diagnosis of syphilis repeat infections.

\section{INTRODUCTION}

Syphilis has re-emerged during the last 15 years as a major public health problem with increasing incidence particularly among men who have sex with men (MSM) in the northern hemisphere. ${ }^{1}$ It is a multistage chronic disease caused by Treponema pallidum

\section{Strengths and limitations of this study}

The clinical utility of IgM detection was investigated for the diagnosis of syphilis and post-treatment follow-up in a prospective cohort of 120 individuals with a new episode of syphilis (72 with repeat infections) and 30 syphilis negative controls.

- Syphilis was diagnosed based on a simultaneous positive treponemal and non-treponemal assay result and/or positive serum PCR targeting polA.

- Sera collected at visit of diagnosis, and 3 and 6 months post-treatment were tested by two different commercially available enzyme immunoassays to detect anti-treponemal IgM, whereby quantitative kinetic decay curves were constructed from the longitudinal quantitative results.

- Specimens collected at diagnosis were also tested for anti-treponemal IgM using a line immunoassay.

- The study population was mostly HIV-infected men who have sex with men taking antiretroviral therapy, therefore the possible effects of HIV infection such as viral load and CD4+ T cell count and gender were not controlled for.

subspecies pallidum, and can be challenging to diagnose, especially very early and repeat infections. Diagnostic strategies remain reliant on serological testing, however, deficiencies in assay performance and persistent anti-treponemal antibody presence following initial infection often hamper diagnostic accuracy. $^{2}$

As the epidemics progress, an increasing proportion of all infections have been noted to be reinfections. ${ }^{3}$ Since reinfections are more likely to present asymptomatically, ${ }^{4}$ timely diagnosis depends on the diagnostic accuracy of serological tests.

Since treponemal tests (TT) remain positive for life, the diagnosis of reinfections typically depends on fourfold or greater rises in non-treponemal test (NTT) titres, such as the rapid plasma reagin (RPR) test. Conversely, a fourfold decline in NTT titres is used to determine the success of syphilis treatment. 
Due to the aspecific nature of NTTs, that relies on antibody binding to lipoidal components released during host cell destruction and also present in low quantities in the $T$. pallidum cell wall, ${ }^{6}$ other biological, infectious or immunological mechanisms can also result in fluctuations in NTT leading to false positive results. ${ }^{7}$

Following an initial infection, immunoglobulin (Ig) M antibodies are the first class of antibodies produced. Early detection of IgM could therefore help with the diagnosis of early syphilis. Current European syphilis guidelines ${ }^{8}$ mention that IgM testing is useful in the assessment of newborns and cerebral spinal fluid. These guidelines also note that a negative IgM result cannot exclude the diagnosis of congenital or neurosyphilis. There is however little published on the clinical utility of IgM testing in these contexts. A study published in $2013^{9}$ evaluated three commercially available IgM enzyme immunoassays (EIAs) using 307 serum samples from individuals with active syphilis. It found that these IgM assays had a median sensitivity of $84.5 \%$ with specificities in the range of $91.4 \%-100 \%$. In $23 / 59(39 \%)$ of cases the IgM EIA test was positive in suspected very early infection where the NTT was negative. Even less has been published in the context of the current outbreaks of syphilis. In many countries a large proportion, and sometimes most syphilis cases, are repeat episodes of syphilis. ${ }^{45} \mathrm{IgM}$ responses are frequently different in repeat episodes of infection. ${ }^{910}$ We could only find one paper that has evaluated the utility of IgM testing for the diagnosis of repeat syphilis. This was a prospective analysis of IgM among a cohort of MSM. It found a low performance of the EIA IgM test for repeat syphilis diagnosis, namely only $38.5 \%$ of repeat syphilis cases were diagnosed correctly with IgM testing. ${ }^{11}$ The number of patients with repeat syphilis in the study was only 13 .

In this study we investigated the clinical utility of $\operatorname{IgM}$ detection in the diagnosis of syphilis and post-treatment follow-up in a prospective cohort of 120 individuals with a new episode of syphilis. In addition, we evaluated the performance of three commercially that is, two EIAs and one line immuno assay (LIA), IgM assays.

\section{MATERIALS AND METHODS}

\section{Study design}

Potentially eligible study participants 18 years or older, in whom a new syphilis diagnosis was made, were consecutively screened and prospectively recruited between January 2014 and August 2015 at a sexually transmitted infection (STI)/HIV clinic in Antwerp, Belgium. Study exclusion criteria were the use of beta-lactam, doxycycline or macrolide antibiotics during the 28 days preceding enrolment. Syphilis diagnosis and disease staging were performed by the study physician according to the Centers for Disease Control guidelines. ${ }^{12}$ Reinfection was defined as an episode of syphilis that followed a previously clearly documented episode of syphilis. ${ }^{3} 13$ Stage-appropriate treatment was administered according to European guidelines. ${ }^{13}$ This involved intramuscular benzathine penicillin 2.4 mu weekly for 3 weeks for late latent syphilis and a stat dose for primary, secondary and early latent syphilis. All participants with syphilis were followed-up by the study team at the STI clinic 3 and 6 months post-treatment. The study physician recorded clinical details and laboratory results in a standardised fashion during each study visit. HIV-infected controls with both negative NTT and TT results were included during the same recruitment period at the same study location. Clinical details were recorded for the controls during a single clinical visit, followed by a same day blood draw.

\section{Patient and public involvement}

Patients or the public were not involved in the design of the study.

\section{Clinical serological testing during routine workout}

Blood was drawn into serum gel tubes (Sarstedt Monovette, Nümbrecht, Germany). Sera were divided and either (1) stored at $4^{\circ} \mathrm{C}-8^{\circ} \mathrm{C}$ until routine syphilis serological testing within 4 days or (2) stored at $-80^{\circ} \mathrm{C}$ within 3 hours for later testing. Routine serological testing included Macro-Vue RPR Card (Becton Dickinson, Sparks, Maryland, USA) and TPA assay (Ortho-Clinical Diagnostics, Rochester, New York, USA) testing following the manufacturer's instructions. Positive RPR results were determined to a titre endpoint.

Sera obtained at baseline were also tested with the SERODIA-T. pallidum particle agglutination (TP-PA) (Fujirebio, Tokyo, Japan) assay and an in-house T. pallidum PCR targeting polA. ${ }^{14}$

\section{EIA and LIA testing for IgM serum antibodies}

In order to investigate if assays from different manufacturers or using another method performed differently and thus would have an impact on our study outcome, we evaluated side by side two IgM EIAs and one LIA.

The anti-T. pallidum IgM EIAs were provided by Euroimmun (Lübeck, Germany) and by Mikrogen GmbH (Neuried, Germany), henceforth referred to as 'EU EIA' and 'MI EIA', respectively. Both assays use microplate wells coated with a mixture of four antigens of T. pallidum: Tp15, Tp17, Tp47 and TmpA. Testing was performed following the manufacturer's instructions (EI_2111M A_UK_C07.doc; V.08/09/2011 and GIRETP011DE.doc; V.April 2010). All quantitative EIA results were expressed in International Units (IU) $/ \mathrm{mL}$. The lower detection limit of the EU EIA was defined as the ratio value of 0.06 by the manufacturer. A ratio result of $\geq 0.8$ to $<1.1$ was defined as borderline. The results were semi-quantitatively evaluated by calculating a ratio of the extinction value of the patient sample over the extinction value of the calibrators. The manufacturer reported a sensitivity and specificity of $100 \%$, with an intra-assay variation (CV) of $4.17 \%$ and inter-assay CV of 5.3\%. With regards to the MI EIA, the manufacturer reported sensitivity was $100 \%$, intra-assay 
$\mathrm{CV} 4.5 \%$ and inter-assay $\mathrm{CV}<11 \%$. Borderline values were defined as $\geq 20-\leq 24 \mathrm{IU} / \mathrm{mL}$.

The recomLine Treponema LIA kit (MI LIA) (Mikrogen $\mathrm{GmbH}$ ) was used for the qualitative determination of the IgM antibodies for baseline samples. This test uses recombinant $T$. pallidum antigens fixed on nitrocellulose membrane strips. Testing was performed according to the manufacturer's instructions (GARLTP002EN, V.2012/08). Objective reading of the strips was done by scanning the strips with a flatbed scanner and analysis software Recomscan (Mikrogen). The density of the antigen was compared with a positive control and ratios were calculated. The assay was reported negative if no antigen was detected (ratio $<1$ ), borderline if only one random antigen was detected (ratio $\geq 1$ ) and positive if at least two random antigens were present (ratio $\geq 1)$. The manufacturer reported sensitivity was $100 \%$ and specificity $95 \%$.

\section{Quality control}

The samples were analysed by a research and diagnostic laboratory unit, both are ISO15189 accredited. One single lot number was used for all EIAs and LIAs. Two laboratory technicians performed the analyses during two batch testing periods of 14 days in 2016. Evaluative testing of the EIAs took place maximum 974 days after the samples of interest were collected. Samples were thawed, tested and refrozen on the same day with a maximum of two freeze thaw cycles.

Due to the prolonged testing period and nature of the routine diagnostic laboratory setting, the reagents used for the TPPA, TPA and RPR tests were from different lot numbers.

The laboratory technicians were blinded from the patient's clinical information and any other syphilis serology result.

Data were manually double entered into the database.

\section{Definitions}

In the absence of a single gold standard test for syphilis, a positive RPR test, or in the case of reinfection a fourfold increase in RPR titre, together with a positive TPPA/ TPA results or a positive T. pallidum PCR test were used to define a new syphilis episode ('syphilis diagnosis').

\section{Statistical analysis}

Percentage agreement and Cohen's kappa $(\kappa)$-coefficient value $^{15}$ were calculated to estimate agreement between the IgM test results. The sensitivity, specificity, positive and negative predictive values with $95 \%$ CIs were calculated using the baseline visit samples. Borderline results for the EIA and LIA testing were included for the first set of analyses as negative (specific scenario) and the second dataset included all borderline results as positive (sensitive scenario).

Continuous variables were expressed as median values and IQR. Associations between categorical variables were assessed with the $\chi^{2}$ test and Fisher's exact test for
Table 1 Study subject characteristics

\begin{tabular}{|c|c|c|}
\hline & $\begin{array}{l}\text { Syphilis-positive } \\
\text { cases } \\
(\mathrm{N}=120)\end{array}$ & $\begin{array}{l}\text { Controls* } \\
(\mathrm{N}=30)\end{array}$ \\
\hline Characteristics & $\begin{array}{l}\text { n (\%)/median } \\
\text { (IQR) }\end{array}$ & $\begin{array}{l}\text { n (\%)/median } \\
\text { (IQR) }\end{array}$ \\
\hline Gender (male) & $119(99)$ & $30(100)$ \\
\hline MSM & $117(98)$ & $24(80) \dagger$ \\
\hline HIV-infected & $103(86)$ & $30(100) \dagger$ \\
\hline Taking ART & $91(88)$ & $24(80)$ \\
\hline $\begin{array}{l}\text { Benzathine penicillin } \\
\text { G treatment }\end{array}$ & $117(98)$ & NA \\
\hline $\begin{array}{l}\text { RPR-C titre at } \\
\text { baseline }\end{array}$ & $64(32-128)$ & 0 \\
\hline Age (years) & $40(31.5-48)$ & $37.5(32-45)$ \\
\hline CD4+ T cell count & $598(448-749)$ & $577(392-684)$ \\
\hline \multicolumn{3}{|c|}{ Syphilis stages and infection history } \\
\hline Syphilis stage & Initial infection $\ddagger$ & $\begin{array}{l}\text { Repeat } \\
\text { infection } \neq\end{array}$ \\
\hline Primary & $12(10)$ & $11(9)$ \\
\hline Secondary & $22(18)$ & $28(23)$ \\
\hline Early latent & $7(6)$ & $24(20)$ \\
\hline Late latent & $7(6)$ & $9(8)$ \\
\hline
\end{tabular}

${ }^{*}$ Control subjects were all HIV positive and non-treponemal and treponemal antibody negative at the time of study inclusion. †Statistically significant difference between syphilis positive group and controls.

$\ddagger$ Percentage calculated with denominator $n=120$ syphilis-positive subject.

ART, antiretroviral therapy; MSM, men who have sex with men; NA, not applicable; RPR, rapid plasma reagin.

small numbers. Mann-Whitney $\mathrm{U}$ test was performed to compare the quantitative results of the IgM assays between initial and repeat syphilis groups, in addition to symptomatic (primary and secondary stage) versus latent syphilis groups. An all-available case approach was used throughout all analyses. Analyses were performed in Stata V.13.1 (StataCorp, College Station, Texas, USA). The statistical significance level was set at 0.05 .

Non-linear models were used to visually assess the decay of the IgM concentrations and RPR titres over time. The exponential decay curves were of the form $\mathrm{Y}=\mathrm{a}^{*} \mathrm{e}^{-\mathrm{b} * \mathrm{X}}$, where $\mathrm{Y}$ was the measurement of the EIA tests and $\mathrm{X}$ the time in months. The exponential decay curves and the graphics were designed using R V.3.4.4 ${ }^{16}$ and the package nlstools. ${ }^{17}$

\section{RESULTS}

\section{Study participants}

In total 150 individuals were included in the study, 120 diagnosed with syphilis and 30 controls. ${ }^{18}$ Study subject characteristics are described in table 1 . Of those who had active syphilis at the time of study enrolment 48/120 
(40\%) presented with a first episode of syphilis (henceforth referred to as 'initial infection') and 72/120 (60\%) presented with a repeat infection. Previous NTT results were available for $107 / 120$, the remaining 13 individuals without previous serological test results were classified as having an initial infection based on the fact that they had never had a diagnosis of or symptoms suggestive of syphilis before. Although initial infections were more often symptomatic $34 / 48(71 \%)$ compared with the repeat infections $39 / 72(54 \%)$, this difference did not reach statistical significance $(\mathrm{p}=0.09) .^{5}$

\section{IgM test results}

The IgM testing was performed on 339, 343 and 150 sera by the EU, MI EIAs and LIA, respectively. Details of the qualitative results are presented in table 2. One sample from a control patient tested IgM positive and another one tested borderline positive with the MI EIA. The LIA also had one false positive and one borderline result. All control samples tested negative with the EU EIA. Slight differences in the number of samples tested per visit (baseline $\mathrm{N}=3$ and $\mathrm{M} 6 \mathrm{~N}=1$ ) between the two EIAs was due to logistical error. Samples were not available for all follow-up visits due to non-attendance; the reason was not recorded.

\section{IgM test performs sub-optimally for diagnosis of syphilis}

The overall diagnostic performance for the two IgM EIAs and LIA assays evaluated was moderate. Table 3 summarises the assay performances in the specific-case scenario. The diagnostic sensitivities of the evaluated assays ranged from $80.9 \%$ to $91.7 \%$ when applied to samples collected at baseline from individuals with initial syphilis. When baseline analyses were stratified per initial or repeat infection, the diagnostic sensitivity decreased significantly in repeat infections for all assays evaluated compared with initial infection $(\mathrm{p}=0.002$ (MI EIA); $\mathrm{p}=0.003$ (EU EIA); $<<0.0001$ (LIA MI)).

The performances of the assay in the sensitive-case scenario are presented in online supplemental table S1.

\section{LIA IgM testing of baseline samples}

The overall performance of the LIA test was moderate (tables 2 and 3). Of the six antigen lines evaluated, Tp47 and TmpA were the most frequently positive. No samples were positive for the marker Tp257. A heat map was created comparing the RPR titre and MI LIA (online supplemental figure S2).

\section{Quantitative assessment of baseline samples with EIA}

When considering the quantitative results of the two EIAs, a significant difference was found between the initial and repeat syphilis groups $(\mathrm{EU} \mathrm{p}=0.0000$; $\mathrm{MI} \mathrm{p}=0.0002)$ with repeat syphilis having lower $\operatorname{IgM}$ concentrations. Moreover, the concentrations were significantly lower for individuals presenting with latent stage syphilis compared with primary and secondary stage (EU EIA $p=0.001$; MI EIA $p=0.0002$ ).
Longitudinal post-treatment follow-up at 3 months and 6 months and kinetic decay curve characteristics of EIA testing At 6 months post-treatment seroreversion of $\operatorname{IgM}$ test results occurred in 57/70 (81\%) samples tested by the EU EIA and 66/90 (73\%) tested by MI EIA. Among the month 6 samples for which a seroreversion was not observed, 6/13 and 7/24 tested with the EU EIA and MI EIA, respectively, did not show a decrease in RPR titre either. Overall, in a total of 19 individuals a decrease in RPR titre post-treatment was not observed. Individuals with an initial syphilis infection demonstrated a faster decrease of almost $30 \%$ in analyte concentration for every additional time unit $(0.55$ (95\% CI: $0.36-$ $0.68)$ ) compared with a repeat syphilis $(0.74$ (95\% CI: 0.62-0.83)) (figure 1). Online supplemental figure S3 represents the decay curves of IgM EIA MI concentration and RPR titres over time.

\section{Agreement between the commercial IgM tests}

The overall agreement was substantial between the two EIA tests when considering all samples that is, samples from baseline, M3 and M6, tested by both assays ( $\mathrm{N}=339)$. The Cohen's kappa was $\kappa: 0.69(85.3 \%)$ and $\kappa: 0.74$ $(86.7 \%)$ for the specific-case and sensitive-case scenarios, respectively.

There was a strong agreement between the LIA and the EIAs: $90.7 \%$ ( $\kappa: 0.81$ ) between the MI EIA and MI LIA, and $95.3 \%$ ( $\kappa: 0.91$ ) between EU EIA and MI LIA in the specific-case scenario. In the sensitive-case scenario the agreement between MI EIA and MI LIA increased further up to $99.3 \%$ ( $\kappa: 0.91$ ) but decreased between EU EIA and MI LIA to $88.7 \%$ ( : 0.77 ).

\section{DISCUSSION}

We aimed to determine whether testing for IgM could aid in the diagnosis of new syphilis infections, including repeat infections, and if it could be useful for posttreatment follow-up. Detection of IgM may help in the diagnosis of syphilis but its diagnostic sensitivity is poor, it was notably low in participants diagnosed with repeat syphilis. Our results are, however, congruent with previous reports of suboptimal IgM test performance overall and for repeat syphilis in particular. ${ }^{11}$ The lower diagnostic sensitivity of IgM in repeat syphilis is compatible with results from other infections where reinfections lead directly to increases in IgG without initial increases in $\operatorname{IgM} .^{10}$

This study represents the most comprehensive evaluation of EIA and LIA IgM testing on serum from individuals with syphilis that we are aware of. We found that both EIA agreed substantially, although that the MI EIA performed in terms of diagnostic sensitivity better than the EU assay. The difference between both sandwich EIAs lays in the pre-treatment of serum with the IgG/ rheumatoid factor (RF) absorbant included in the EIA reagent kit of EU. The absorbant removes the antibodies of the IgG classes and the RFs, known to be possible 


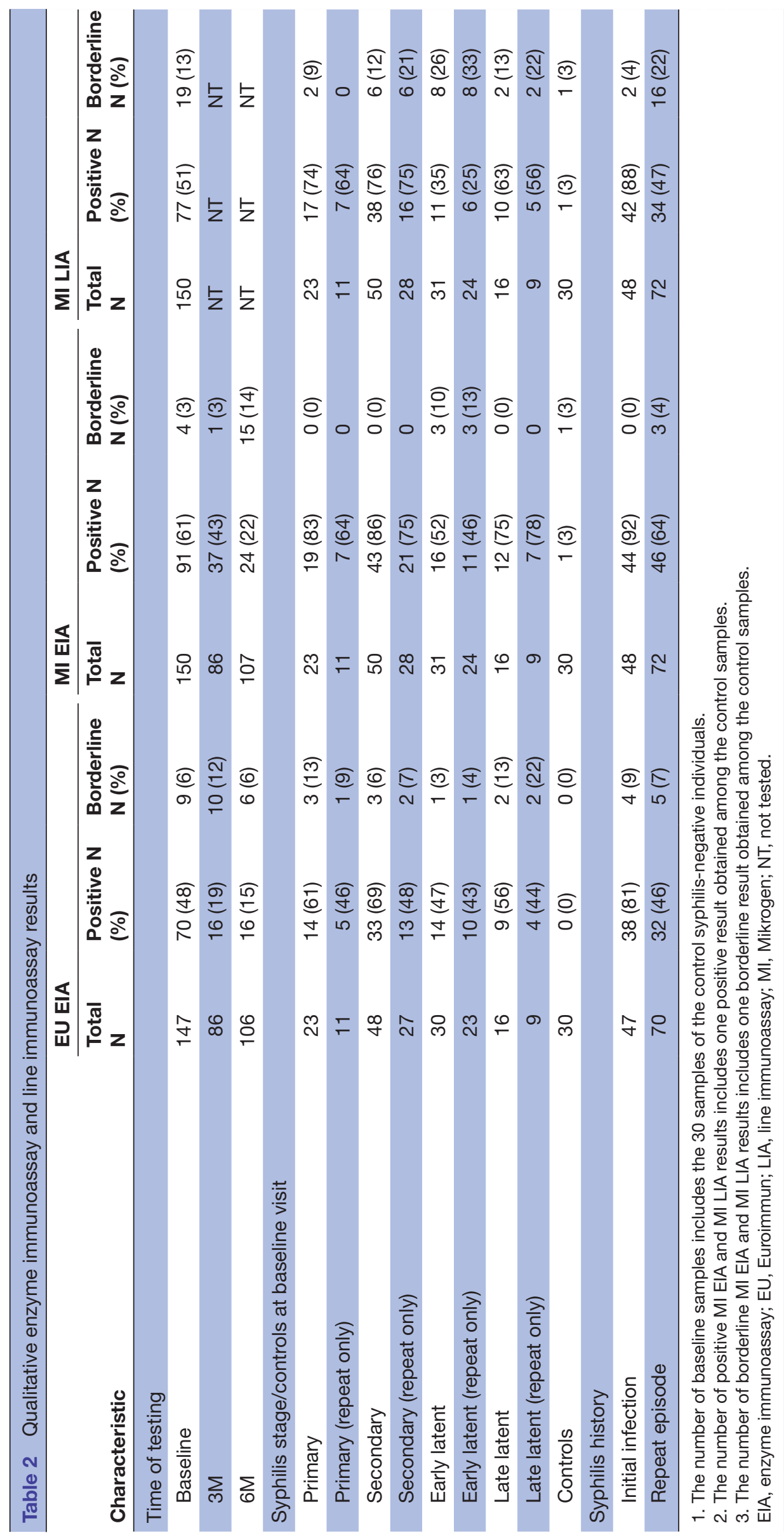


Table 3 Cross-tabulations and performance characteristics of the serological assays (EIAs/LIA) tested in this study according to the specific scenario*

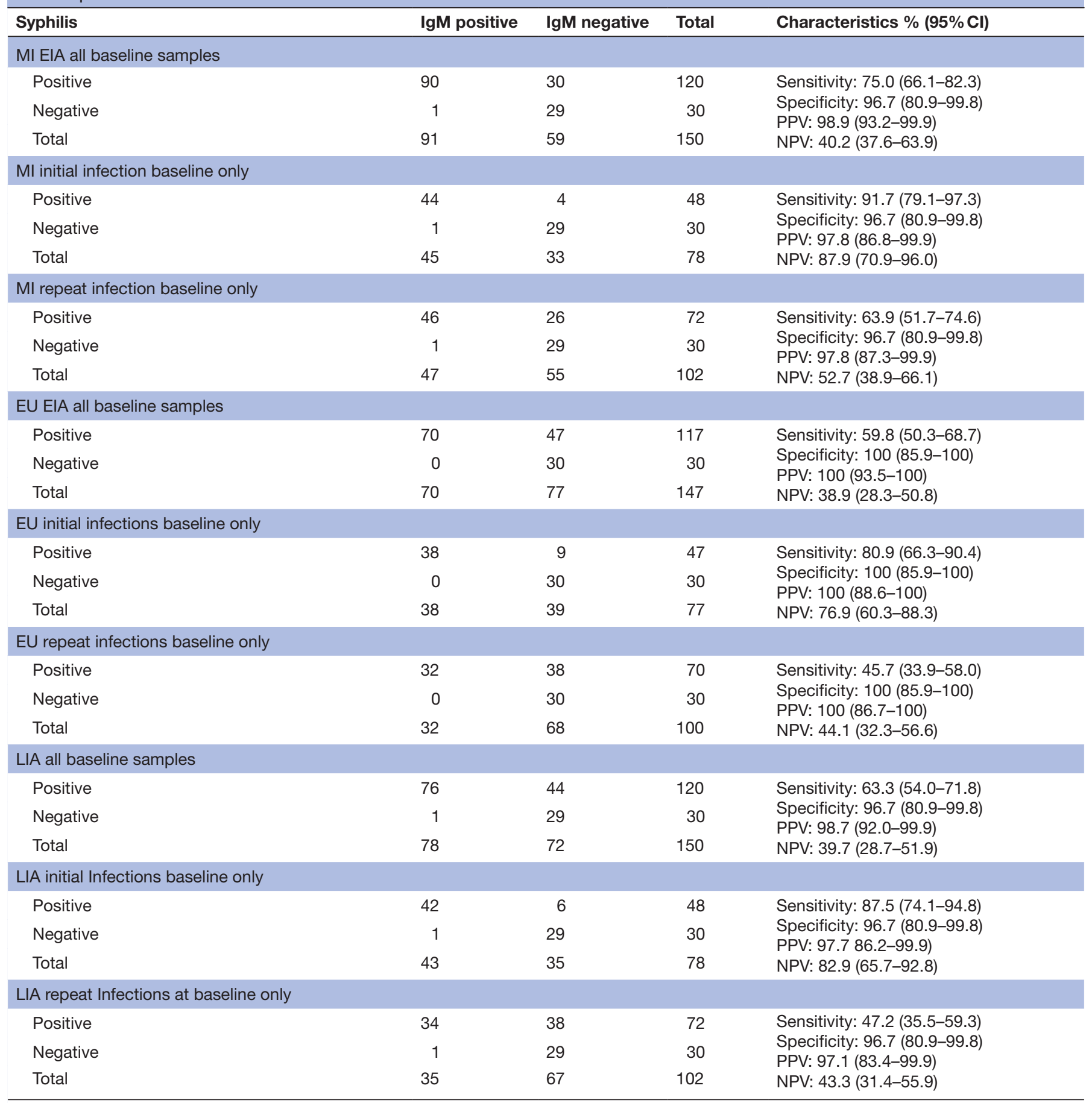

${ }^{*}$ Borderline results included as negative for all values.

EIA, enzyme immunoassay; EU, Euroimmun; LIA, line immunoassay; MI, Mikrogen; NPV, negative predictive value; ; PPV, positive predictive value.

interferences in IgM EIA. False positive reactions may occur in pathogen-specific IgM detection when the IgM RFs bind to IgG immune complexes. On the other hand, false negative results may appear when pathogen-specific IgM antibodies are displaced by stronger binding IgG. ${ }^{19}$ We cannot rule out that this extra IgG/RF removal step may have contributed to the differences in test performance found between both EIAs.
The general performance of the LIA for the baseline syphilis diagnosis was similar to the EIAs. The antigen line reaction varied by sample and in some samples only a few antigens could be demonstrated. Since no particular single antigen or line can be attributed to a stage or profile, an overall qualitative result should be considered.

In the majority of the IgM positive cases detected at baseline, the IgM antibodies disappeared 6 months after 


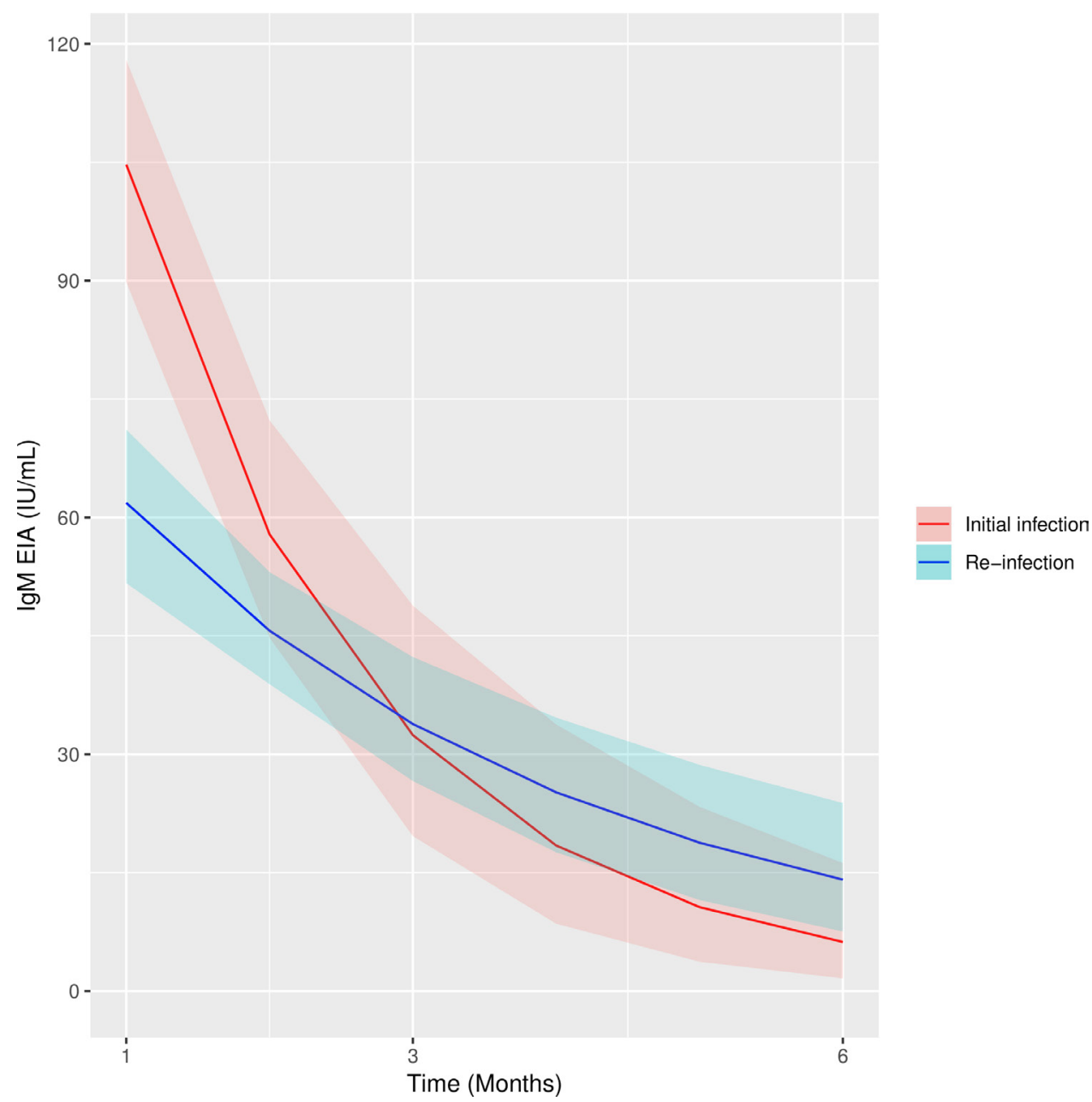

Figure 1 Initial and post-treatment decay curves of recomWell EIA IgM. EIA, enzyme immunoassay.

treatment. Interestingly, the decline in IgM concentrations occurred faster in initial syphilis compared with repeat syphilis. Our study hints but remains inconclusive and additional studies are required to investigate whether IgM concentration measurements may be a more objective, useful and high throughput method compared with RPR for the follow-up of syphilis treatment.

More studies are needed to investigate the role of IgM in syphilis diagnosis on a more diversified and larger scale (eg, women, non-MSM populations). Since our study only included individuals with a dual positive NTT/TT we were unable to evaluate if IgM values might precede NTT/TT seroconversion. A previous study did find that IgM could be useful in the early diagnosis of syphilis in the subset of patients with equivocal TT and negative NTTs. ${ }^{9}$

Strengths of this study include the comprehensive characterisation of sera and its prospective nature. Limitations to this study include the fact that the study population was mostly HIV infected. We did not control for possible effects of HIV infection such as viral load and CD4+ T cell count, although notably most HIV-infected participants were taking antiretroviral therapy and had a high immune cell count thus the likelihood of this having an effect on the test outcome is unlikely. The study was not designed to adequately evaluate the assays' specificity. ${ }^{20}$ Previous serology results were not available for 13 patients. For this group syphilis history was reliant on patient recall of clinical symptoms. It is possible that some individuals unknowingly had a previous syphilis episode, therefore the number of reinfections could be higher than reported. This could possibly affect IgM performance results. Moreover, follow-up sera samples were not available for all $3 \mathrm{M}$ and $6 \mathrm{M}$ study participants, however, most (88\%) endpoint M6 samples were analysed.

\section{CONCLUSIONS}

In conclusion, the overall diagnostic performance for the two IgM EIAs and LIA assays evaluated was moderate. When IgM results were stratified per initial or repeat infection, the diagnostic sensitivity decreased significantly in repeat infections for all assays evaluated. This could lead to missed infections. Individuals with repeat syphilis or presenting with latent stage syphilis had lower IgM concentrations compared with initial infection and primary/secondary stage syphilis, respectively. Future research could evaluate the utility of IgM in the follow-up of treatment. For example, serial IgM testing may play a role in the management of serofast syphilis. 
Acknowledgements We would like to thank the ITM laboratory teams for their involvement in the study and the authors gratefully acknowledge the individuals who participated in this study. Mikrogen and Euroimmun generously provided the tests used in this study. Parts of this work have been presented at IUSTI and ECCMID conferences.

Contributors KKO, IDB, MVE, CRK and TC conceived the study. HS and KKO coordinated and performed laboratory analyses and entered data. MVE, IDB and TC supervised laboratory activities. KKO and AT managed and conducted the data analyses. KKO wrote the first draft. All authors contributed to the final version of the manuscript and approved the final manuscript.

Funding This work was part of Project ID: 757003 funded by the Flemish Government-Department of Economy, Science \& Innovation granted to CRK. The anti-Treponema pallidum IgM assays were provided free of charge by Euroimmun (Lübeck, Germany) and by Mikrogen GmbH (Neuried, Germany).

Competing interests None declared.

Patient and public involvement Patients and/or the public were not involved in the design, or conduct, or reporting, or dissemination plans of this research.

Patient consent for publication Not required.

Ethics approval The Institutional Review Board of the ITM and the Ethics Committee of the University Hospital Antwerp approved this study (13/44/426). Written informed consent for study participation and reporting of anonymised clinical details was obtained from all participants upon study inclusion.

Provenance and peer review Not commissioned; externally peer reviewed.

Data availability statement Data are available upon reasonable request. The data supporting the findings of this publication are retained at the Institute of Tropical Medicine (ITM), Antwerp and will not be made openly accessible due to ethical and privacy concerns. According to the ITM research data sharing policy, only fully anonymised data can be shared publicly. The data are de-identified (using participant identification numbers only) but not fully anonymised and it is not possible to fully anonymise them due to the longitudinal nature of the data. Data can however be made available after approval of a motivated and written request to the ITM at ITMresearchdataaccess@itg.be. The ITM data access committee will verify if the dataset is suitable for obtaining the study objective and assure that confidentiality and ethical requirements are in place.

Open access This is an open access article distributed in accordance with the Creative Commons Attribution Non Commercial (CC BY-NC 4.0) license, which permits others to distribute, remix, adapt, build upon this work non-commercially, and license their derivative works on different terms, provided the original work is properly cited, appropriate credit is given, any changes made indicated, and the use is non-commercial. See: http://creativecommons.org/licenses/by-nc/4.0/.

\section{ORCID iDs}

Irith De Baetselier http://orcid.org/0000-0002-1804-252X

Tania Crucitti http://orcid.org/0000-0002-2235-6038

\section{REFERENCES}

1 Abara WE, Hess KL, Neblett Fanfair R, et al. Syphilis trends among men who have sex with men in the United States and Western
Europe: a systematic review of trend studies published between 2004 and 2015. PLoS One 2016;11:e0159309.

2 Jost H, Castro A, Cox D, et al. A comparison of the analytical level of agreement of nine treponemal assays for syphilis and possible implications for screening algorithms. BMJ Open 2013;3:e003347.

3 Kenyon C, Lynen L, Florence E, et al. Syphilis reinfections pose problems for syphilis diagnosis in Antwerp, Belgium - 1992 to 2012. Euro Surveill 2014;19:20958.

4 Kenyon C, Osbak KK, Apers L. Repeat syphilis is more likely to be asymptomatic in HIV-infected individuals: a retrospective cohort analysis with important implications for screening. Open Forum Infect Dis 2018;5:ofy096.

5 Kenyon C, Tsoumanis A, Osbak K, et al. Repeat syphilis has a different immune response compared with initial syphilis: an analysis of biomarker kinetics in two cohorts. Sex Transm Infect 2018;94:180-6.

6 Borkhardt HL, Zielinski S. Influence of cardiolipin antibodies on the binding of treponemal specific antibodies in the fluorescence treponemal antibody absorption test and the Treponema pallidum immobilisation test. J Med Microbiol 1997;46:965-72.

7 Nandwani R, Evans DT. Are you sure it's syphilis? A review of false positive serology. Int J STD AIDS 1995;6:241-8.

8 Janier M, Hegyi V, Dupin N, et al. European guideline on the management of syphilis. J Eur Acad Dermatology Venereol 2014;2014:1581-93.

9 Bosshard PP. Usefulness of IgM-specific enzyme immunoassays for serodiagnosis of syphilis: comparative evaluation of three different assays. J Infect 2013;67:35-42.

10 Landry ML. Immunoglobulin M for acute infection: true or false? Clin Vaccine Immunol 2016;23:540-5.

11 Pastuszczak M, Kotnis-Gąska A, Jakubowicz B, et al. Utility of antitreponemal IgM testing in the diagnosis of early and repeat syphilis among HIV-infected and non-infected patients. Int J STD AIDS 2018:29:890-4.

12 Workowski KA, Berman S. Centers for disease control and prevention (CDC). sexually transmitted diseases treatment guidelines, 2010. MMWR Recomm reports Morb Mortal Wkly report Recomm reports 2010;59:1-110.

13 French P, Gomberg M, Janier M, et al. IUSTI: 2008 European guidelines on the management of syphilis. Int J STD AIDS 2009;20:300-9.

14 Liu H, Rodes B, Chen CY, et al. New tests for syphilis: rational design of a PCR method for detection of Treponema pallidum in clinical specimens using unique regions of the DNA polymerase I gene. $J$ Clin Microbiol 2001;39:1941-6.

15 Cohen J. A coefficient of agreement for nominal scales. Educ Psychol Meas 1960;20:37-46.

16 Team RC. R: a language and environment for statistical computing 2018.

17 Baty F, Ritz C, Charles S, et al. A Toolbox for Nonlinear Regression in $R$ : The Package nlstools. J Stat Softw 2015;66:1-21.

18 Osbak K, Abdellati S, Tsoumanis A, et al. Evaluation of an automated quantitative latex immunoturbidimetric non-treponemal assay for diagnosis and follow-up of syphilis: a prospective cohort study. $J$ Med Microbiol 2017;66:1130-9.

19 Martins TB, Jaskowski TD, Mouritsen CL, et al. An evaluation of the effectiveness of three immunoglobulin G (lgG) removal procedures for routine IgM serological testing. Clin Diagn Lab Immunol 1995;2:98-103.

20 Series TS, Assessment D. Diagnostic assessment TSS-6 syphilis rapid diagnostic tests. Geneva World Health Organisation, 2018. 